\title{
Global fecal microRNA profiling in the identification of biomarkers for colorectal cancer screening among Asians
}

\author{
LEE CHENG PHUA ${ }^{1}$, XIU PING CHUE ${ }^{1}$, POH KOON KOH ${ }^{2}$, \\ PEH YEAN CHEAH ${ }^{2,3,4}$, ERIC CHUN YONG CHAN ${ }^{1}$ and HAN KIAT HO ${ }^{1}$
}

\author{
${ }^{1}$ Department of Pharmacy, Faculty of Science, National University of Singapore, Singapore 117543; \\ ${ }^{2}$ Department of Colorectal Surgery, Singapore General Hospital, Singapore 169856; ${ }^{3}$ Saw Swee Hock \\ School of Public Health, National University of Singapore, Singapore 117597; ${ }^{4}$ Duke-NUS Graduate \\ Medical School, National University of Singapore, Singapore 169857, Republic of Singapore
}

Received January 29, 2014; Accepted March 14, 2014

DOI: $10.3892 /$ or.2014.3193

\begin{abstract}
Fecal microRNAs (miRNAs) are increasingly explored as non-invasive markers of colorectal cancer (CRC). However, its holistic profile in Asian CRC patients remains elusive. In the present study, the global human fecal miRNAs in Asian Chinese CRC patients was assayed to elucidate novel diagnostic fecal markers. Additionally, the influence of blood in stool on fecal miRNA levels was investigated for the first time. Microarray analysis was applied to profile the fecal miRNAs extracted from CRC patients and healthy subjects. Concurrently, surgically resected tumor and matched normal mucosae were analyzed. Potential fecal miRNA markers were further confirmed using real-time PCR in 17 CRC patients and 28 healthy subjects. Global miRNA profiling uncovered 17 fecal markers $(\mathrm{P}<0.05)$ differentially regulated in $\mathrm{CRC}$. Fecal miR-223 and miR-451 represented robust markers in distinguishing CRC patients from healthy subjects, as evident from areas under the receiver operator characteristic curves of 0.939 and 0.971, respectively. Blood in stool affected fecal miR-451, miR-223 and miR-135b levels to a varying extent and substantially impacted the interpretation of the clinical data. Notably, a discrete set of aberrant miRNAs occurred within the tumor, indicating the presence of contributors beyond the exfoliation of tumor cells to the fecal miRNA profile. In summary, the utility of a global miRNA screening approach was successfully demonstrated in elucidating diagnostic markers of CRC. In particular, fecal miR-223 and miR-451 hold promise in detecting CRC.
\end{abstract}

Correspondence to: Dr Han Kiat Ho or Professor Eric Chun Yong Chan, Department of Pharmacy, Faculty of Science, National University of Singapore, 18 Science Drive 4, Singapore 117543, Republic of Singapore

E-mail: phahohk@nus.edu.sg

E-mail: phaccye@nus.edu.sg

Key words: colorectal cancer, feces, microRNA, blood in stool

\section{Introduction}

Colorectal cancer (CRC) is the third most common cancer in men and the second in women worldwide and accounts for almost $8 \%$ of all cancer-related deaths (1). In Asian countries including China, Singapore, South Korea and Japan, CRC has displayed a rapidly rising trend (2). A 2- to 4-fold upsurge in the incidence of CRC has been encountered over the past few decades (3). In order to abate disease mortality, timely detection of CRC by population screening has been a pivotal strategy. However, existing screening tools such as fecal occult blood test (FOBT) or colonoscopy either lack sensitivity and specificity (4) or are too invasive and costly. Fecal DNA (5) and mRNA testing (6) are emerging non-invasive techniques, but their wide acceptance awaits further optimization and validation with respect to the clinical diagnostic value. These limitations have spawned efforts to develop an alternative fecal-based method that is accurate yet easily administered as a population screening tool for CRC.

As prevailing studies focused on DNA and mRNA aberrations as potential fecal markers, the role of post-transcriptional changes remains underexplored. MicroRNAs (miRNAs) are 19-24 nucleotide non-coding RNAs which negatively regulate gene expression post-transcriptionally by suppressing translation and facilitating degradation of target mRNAs (7). Due to their ability to modulate the expression of oncogenes and tumor suppressors directly, miRNAs have increasingly been implicated in tumorigenesis (8-11). A growing number of clinical studies have congruently shown deregulated miRNA expression in surgically excised colorectal tumor, for instance an upregulation of oncogenic miR-135b that inhibits the tumor suppressor adenomatous polyposis coli (APC) gene, compared to matched normal mucosae (12-20). Owing to its direct contact with the colorectum, feces represent a valuable resource of this unique miRNA signature for the detection of CRC. It was hence hypothesized that fecal miRNAs could serve as candidate markers for the non-invasive screening of CRC.

While targeted profiling of selected fecal miRNAs have been explored (21-26), a holistic understanding of the global fecal miRNA profile in CRC patients is lacking, particularly 
in Asian patients. Non-targeted fecal miRNA profiling enables the discovery of novel diagnostic markers but has hitherto been conducted predominantly in Caucasian CRC patients $(27,28)$ who may possess different genetic predispositions $(3,29)$ and hence dissimilar fecal miRNA signature from their Asian counterparts. A global profiling study is, therefore, warranted in the Asian context. Furthermore, it remains to be determined if the global miRNA profile characterizing the feces of CRC patients reflects, or differs from, that within the colorectal tumor. In addition, gut bleeding is a clinically prevalent phenomenon of CRC. With the significant presence of miRNAs in the plasma, platelets and blood cells $(30,31)$, the potential impact of blood in stool on the fecal miRNA profile needs to be addressed. To date, this pertinent factor that influences the interpretation of fecal miRNA data in disease biomarker profiling remains unclear.

In the present study, the global human fecal miRNA profiles were characterized in CRC patients and healthy subjects in Singapore to elucidate fecal markers for non-invasive detection of the disease. Independently, tissue miRNA profiles were also characterized and compared between cancer and matched normal mucosae resected from the same cohort of CRC patients. The effect of blood in stool on the levels of fecal miRNA markers was investigated for the first time.

\section{Materials and methods}

Clinical population and samples. CRC patients and healthy subjects were recruited at the Outpatient Specialist Clinic at the Singapore General Hospital from September 2011 to February 2013. All subjects were Han Chinese. The inclusion criterion for CRC patients was the diagnosis of sporadic CRC where surgical tumor resection had not been performed. Exclusion criterion was administration of neoadjuvant chemotherapy or radiotherapy. Randomly selected healthy subjects were eligible for the present study if they had undergone a colonoscopy within the past 5 months to confirm the absence of CRC or pre-cancerous polyps. Exclusion criteria comprised a history of CRC, a history of polyps within the last 3 years, the presence of inherited CRC syndromes (such as, Lynch syndrome and familial adenomatous polyposis) and a family history of hereditary CRC. Subjects in both groups did not have co-existent inflammatory bowel disease. The present study was endorsed by the Institutional Review Board at the Singapore General Hospital (2010/042/B) and all subjects provided written informed consent prior to participation in the study.

Fecal samples were collected domestically by CRC patients before surgical resection and by healthy subjects within 1 month of enrolment in the study. Detailed instructions for sample collection were provided to all subjects. Briefly, a representative aliquot of feces was collected in a sealed plastic container. Care was taken to prevent contact with urine and toilet water. The samples were transported to the hospital on the same day, or were otherwise kept at $4^{\circ} \mathrm{C}$ and transferred to the hospital within 2 days. All samples were stored at $-80^{\circ} \mathrm{C}$ immediately upon receipt at the hospital.

Surgical tissues resected from the same cohort of CRC patients were snap-frozen in liquid nitrogen, microdissected and stored at $-80^{\circ} \mathrm{C}$ until analysis. Careful microdissection of the excised tissue ensured that at least $90 \%$ of the tumor specimen consisted of cancer cells. Matched normal mucosa samples were obtained from excised tissue at least $5 \mathrm{~cm}$ away from the edges of the tumor. Routine histological evaluation of the tumor specimens was also conducted by a blinded pathologist to determine the stage and grade of the cancer.

Study design. The study was implemented in 2 phases, namely phase I, biomarker screening, and phase II, biomarker confirmation. In phase I, biomarker screening, global profiling by microarray was performed on the fecal miRNAs extracted from $8 \mathrm{CRC}$ (stage B-C) patients and 8 healthy subjects. Concurrently, the platform was employed to compare the miRNA profiles of tumors $(n=8)$ and paired normal mucosa $(n=8)$ surgically excised from the same cohort of CRC patients. The differential miRNA expression patterns established from the fecal and tissue analyses were compared.

In phase II, biomarker confirmation, selected fecal markers identified from phase I were confirmed by real-time PCR. In phase IIa, the validation of the real-time PCR approach to biomarker confirmation was performed using miR-135b, an established fecal marker for CRC $(22,27,28)$, on a randomly chosen subset of 9 CRC patients and 19 healthy subjects. In phase IIb, the CRC-related dysregulation of selected fecal miRNAs shown in phase I was confirmed on an extended cohort of CRC patients $(n=17)$ and healthy subjects $(n=28)$. The robustness of these markers in discriminating CRC subjects from healthy subjects was evaluated.

Materials. MirVana ${ }^{\mathrm{TM}}$ miRNA isolation kit was purchased from Ambion. TaqMan ${ }^{\circledR}$ MicroRNA Assays, Taqman ${ }^{\circledR}$ microRNA RT kit and Taqman ${ }^{\circledR}$ Universal PCR Master Mix (2X) with no AmpErase UNG were products of Applied Biosystems. QIAshredder was supplied by Qiagen. Nucleasefree water was obtained from Invitrogen.

Total RNA extraction from feces and colorectal tissue. Total RNA (including miRNAs) was isolated from $\sim 50 \mathrm{mg}$ of frozen feces (sampled from 3 different points of the fecal mass) or $20 \mathrm{mg}$ of fresh frozen tissue using the mirVana ${ }^{\mathrm{TM}}$ miRNA isolation kit. Briefly, the samples were homogenized by plastic pestle on ice in $600 \mu \mathrm{l}$ of lysis buffer. The homogenate was passed through QIAshredder and centrifuged at 18,000 x g for $2 \mathrm{~min}$. Subsequent steps were performed in accordance with the manufacturer's instructions. Total RNA was then quantified using NanoDrop (Thermo Scientific). Quality of the RNA was determined using Agilent bioanalyzer 2100 (Agilent Technologies).

\section{Microarray-based profiling (phase I)}

Labeling and hydridization. Microarray analysis was performed on Agilent Human miRNA 8x60K format v16 (based on Sanger miRbase version 16.0) by Genomax Technologies. Each array contained probes interrogating 1,347 miRNAs. In the analysis, miRNAs were labeled with Agilent miRNA Complete Labelling and Hyb kit. Briefly, total RNA (100 ng) was phosphorylated with Calf Intestinal Alkaline Phosphatase before ligating with cyanine3-pCp by T4 RNA Ligase. Labeled RNA was dried completely using vacuum centrifugation and reconstituted in $17 \mu \mathrm{l}$ of nuclease-free water and hybridized onto Agilent SurePrint G3 Human miRNA 
$8 \times 60 \mathrm{~K}$ microarray for $20 \mathrm{~h}$ at $55^{\circ} \mathrm{C}$. After hybridization, the microarray slide was washed before scanning on the Agilent High Resolution Microarray Scanner (C-model). Raw signal data were extracted with Agilent Feature Extraction Software (version 10.7.1.1).

Data pre-processing. Data was pre-processed by logarithmic transformation and normalization to the 90th percentile. Only miRNAs present in at least $75 \%$ of samples in any 1 out of 2 conditions, at raw signal intensities $>20$, were analyzed.

Selection of endogenous controls for real-time PCR. To the processed data from the fecal miRNA microarray, the following cut-offs were applied: i) small coefficient of variation (CV) of $<25 \%$ across samples, ii) minimal fold-change in the range of $0.8-1.25$ between groups, and 3 ) raw signal intensity $>100$. Selection of appropriate endogenous control was carried out based on the commercial availability of Taqman ${ }^{\circledR}$ real-time PCR assays and the absence of publications on their dysregulation in CRC. Finally, candidate endogenous controls were verified on real-time PCR. GeNorm Algorithm was utilized to determine $M$ value, a measure of expression stability (32). Suitable endogenous controls have $\mathrm{M}$ values $<1.5$.

Real-time PCR-based profiling (phase II). Primers for reverse transcription (RT) and real-time PCR were provided by TaqMan ${ }^{\circledR}$ MicroRNA assays. The assays target miR-223 (assay ID: 002295), miR-451 (assay ID: 001141), miR-135b (assay ID: 002261), miR-1202 (assay ID: 002858), miR-4257 (assay ID: 244369) and miR-3937 (assay ID: 462743). Total RNA (10 ng) was reverse-transcribed using Taqman ${ }^{\circledR}$ microRNA RT kit, according to the manufacturer's instructions. The PCR reaction mix contained $1.33 \mu \mathrm{l}$ of cDNA, $1 \mu \mathrm{l}$ of primers, $10 \mu \mathrm{l}$ of Taqman $^{\circledR}$ Universal PCR Master Mix (2X) with no AmpErase UNG and $7.67 \mu \mathrm{l}$ of nuclease-free water. The thermal cycling procedure encompassed pre-cycling heat activation at $95^{\circ} \mathrm{C}$ for $10 \mathrm{~min}$, followed by 40 cycles of denaturation at $95^{\circ} \mathrm{C}$ for $15 \mathrm{sec}$ and annealing/extension at $60^{\circ} \mathrm{C}$ for $60 \mathrm{sec}$, in a CFX96 real-time PCR system (Bio-Rad). Reactions were performed in triplicates. Data were obtained as average $\mathrm{Ct}$ values, and normalized against endogenous controls as $\Delta \mathrm{Ct}$.

Validation of real-time PCR method for clinical profiling. The amplification efficiencies of 3 target miRNAs (miR-223, miR-451 and miR-135b) and 2 endogenous controls (miR-1202 and miR-4257) were examined. In the PCR reaction, primers were added to five different cDNA concentrations that were serially diluted. The generated $\mathrm{Ct}$ values were then plotted against the logarithm of cDNA concentrations. Amplification efficiency was derived from the slope of the log-linear portion of the calibration curve (efficiency $=10^{-1 / \text { slope }}-1$ ). No-templatecontrol (NTC) and no-reverse-transcription (NRT) control were included.

Effect of blood on the fecal miRNA levels. Feces (Bristol stool scale type 4) were collected from a healthy subject who was tested negative by FOBT. Blood was spiked into feces to simulate different clinical levels of blood in stool, namely $0.1 \mathrm{mg}$ $\mathrm{Hb} / \mathrm{g}$ stool (occult), $1 \mathrm{mg} \mathrm{Hb} / \mathrm{g}$ stool (occult), $10 \mathrm{mg} \mathrm{Hb} / \mathrm{g}$ stool and $100 \mathrm{mg} \mathrm{Hb} / \mathrm{g}$ stool (gross). Unspiked neat feces and blood served as controls. The experiment was performed in triplicates. RNA was isolated, reverse-transcribed and analyzed by real-time PCR as detailed above. The normalized levels of $\mathrm{miR}-451$, miR-223 and $\mathrm{miR}-135 \mathrm{~b}$ were expressed as a ratio with respect to neat feces.

Statistical analysis. In microarray-based profiling, data preprocessing, filtering, univariate t-test and fold-change analysis were performed using GeneSpring GX 11.5. Specifically, independent samples t-test was used to compare miRNAs from fecal samples of CRC patients and healthy subjects, while paired samples t-test was used to compare miRNAs from tumor tissue and matched normal mucosae. Multivariate partial least-squares discriminant analysis (PLS-DA) was performed using SIMCA-P version 11.0 software (Umetrics). The validity of the PLS-DA model was ascertained by response permutation testing (100 repetitions). A list of differential miRNAs was identified based on variable importance for projection (VIP) score of $>1.2$ in the PLS-DA coupled to P-value of $<0.05$ in the t-tests. Heat map was generated using R (www.r-project.org).

In real-time PCR-based profiling, univariate analysis of relative levels of miRNAs was accomplished using the Relative

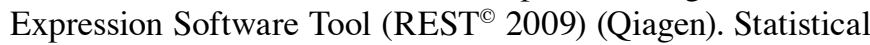
significance was established at $\mathrm{P}<0.05$. Multivariate logistic regression was performed using MedCalc version 12.7 using $\Delta \mathrm{Ct}$ values. To evaluate the robustness of the markers in classifying CRC patients and healthy individuals, ROC analyses were performed using the predicted Y-values from the regression model. Corresponding areas under the ROC curve (AUC) were determined.

\section{Results}

Patient characteristics. A total of 45 participants including 17 CRC patients and 28 healthy subjects were recruited. The clinical characteristics of the participants are summarized in Table I. Notably, there was an uneven distribution of age $(\mathrm{P}<0.005$, independent samples $\mathrm{t}$-test $)$ and gender $(\mathrm{P}<0.01$, $\chi^{2}$ test) between the groups. These factors were hence adjusted in subsequent analysis. Among the CRC patients, the cancer stages ranged from Dukes' stages B to D. Most tumors were moderately differentiated.

Elucidation of CRC-related miRNA profiles in feces and tissue (phase I). In the microarray-based fecal miRNA expression analysis, $277 \mathrm{miRNAs}$ were detected after the exclusion of low or non-uniform signals. Univariate tests uncovered 17 human fecal miRNA markers characterizing CRC $(\mathrm{P}<0.05)$, although multivariate analysis did not differentiate CRC patients from healthy subjects. Fig. 1A shows the heat map of these 17 fecal miRNAs and their associated fold-changes relative to healthy subjects. Among the fecal miRNAs, miR-451 was the most prominently dysregulated.

In the global tissue miRNA profiling, 287 miRNAs were detected. Tumor miRNA signature was clearly distinct from matched normal mucosa as shown in the PLS-DA scores plot (3 latent variables, $R^{2} X=0.619, R^{2} Y=0.992, Q^{2}=0.883$; Fig. 1B). Based on PLS-DA, 79 discriminating human miRNAs were uncovered $(\mathrm{VIP}>1.2, \mathrm{P}<0.05)$ and summarized as a heat map in Fig. 1C. 
A

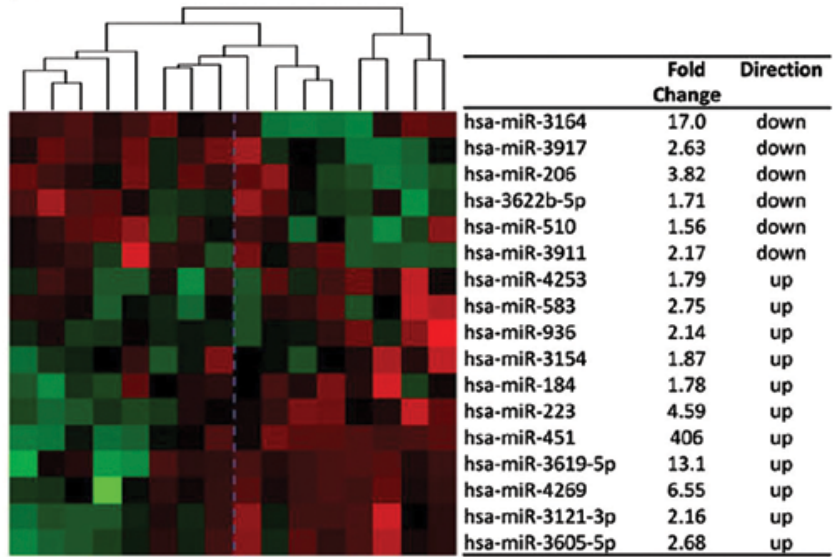

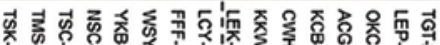

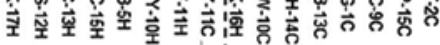

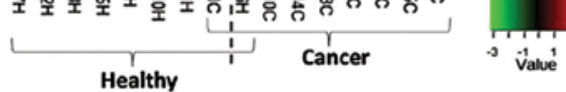

B

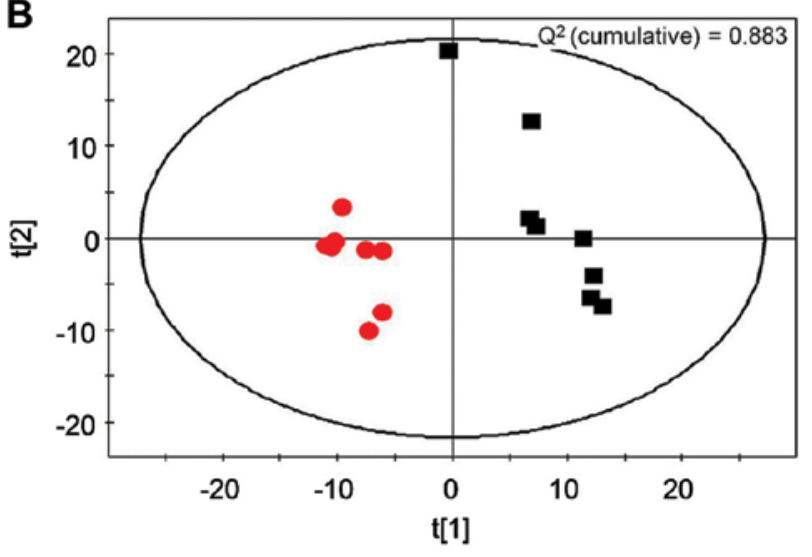

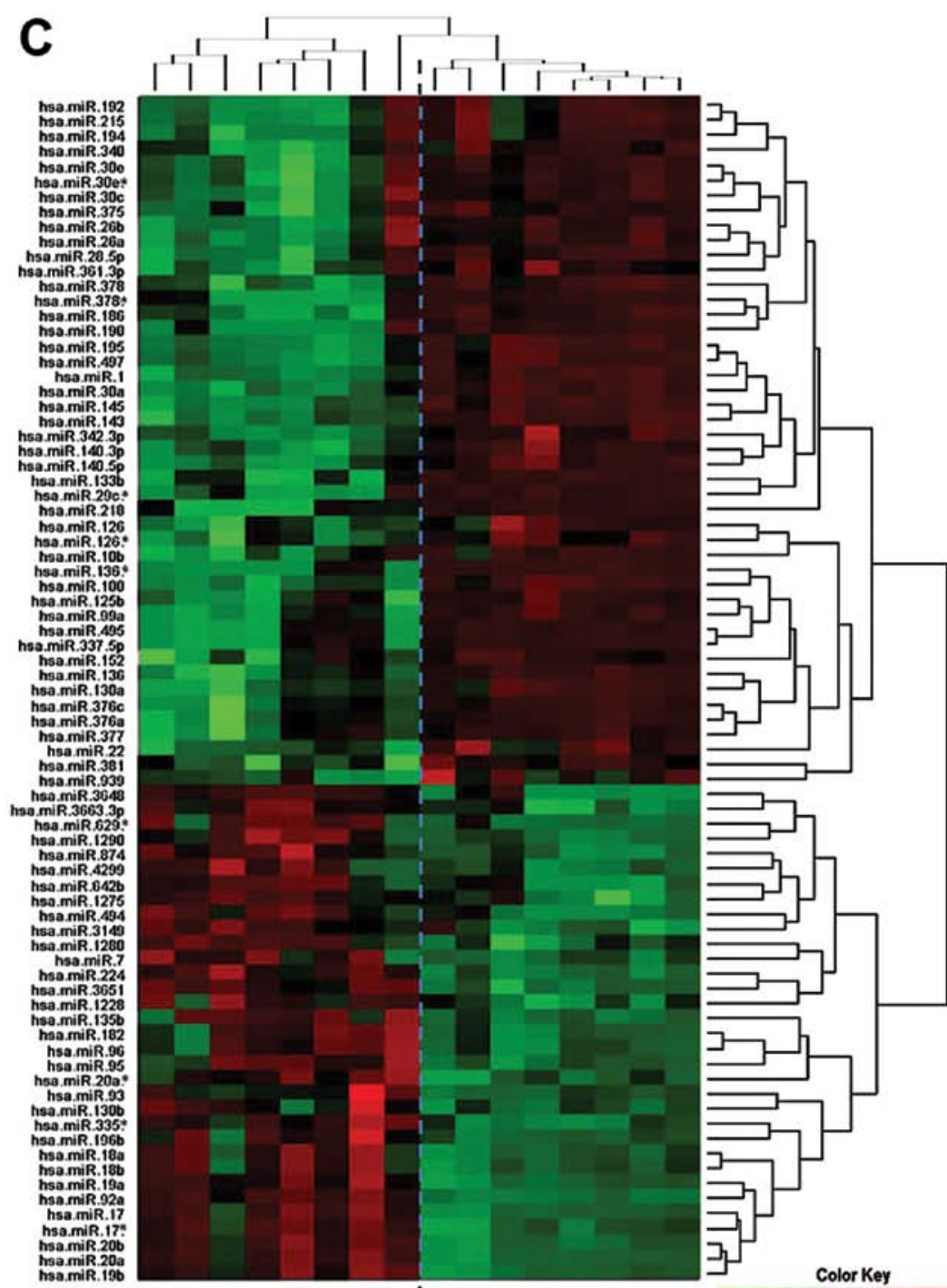

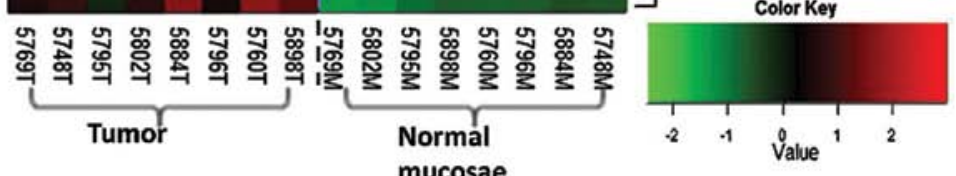

Figure 1. Fecal and tissue miRNA markers characterizing CRC. (A) Heat map showing 17 fecal miRNAs characterizing CRC $(\mathrm{P}<0.05)$ and their associated fold changes and directions of change (relative to healthy subjects). Heat map was built based on processed array signals. (B) PLS-DA scores plot differenti-

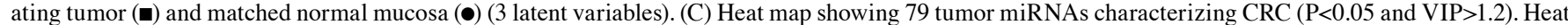
map was built based on processed array signals. 
Table I. Clinical characteristics of CRC patients and healthy subjects.

\begin{tabular}{lcc}
\hline Characteristics & $\begin{array}{c}\text { CRC patients } \\
(\mathrm{n}=17)\end{array}$ & $\begin{array}{c}\text { Healthy subjects } \\
(\mathrm{n}=28)\end{array}$ \\
\hline Age (years) & & \\
Mean & 63.7 & 55.1 \\
Range & $46-80$ & $36-79$ \\
Gender, n (\%) & & \\
Male & $13(76.5)$ & $10(35.7)$ \\
Female & $4(23.5)$ & $18(64.3)$ \\
Tumor site, $\mathrm{n}(\%)$ & & \\
Ascending colon & $1(5.9)$ & \\
Sigmoid & $4(23.5)$ & \\
Rectosigmoid & $3(17.6)$ & \\
Rectum & $9(52.9)$ & \\
Dukes' stage, $\mathrm{n}(\%)$ & & \\
B & $8(47.1)$ & \\
C & $7(41.2)$ & \\
D & $2(11.8)$ & \\
Grade & & \\
Well differentiated & $2(11.8)$ & \\
Moderately differentiated & $14(82.4)$ & \\
Poorly differentiated & $1(5.9)$ & \\
\hline
\end{tabular}

Candidate endogenous controls for real-time PCR. In addition to uncovering differentially expressed miRNAs, a further capability of microarray analysis was to identify consistently expressed fecal miRNAs that may serve as normalizers on subsequent real-time PCR analysis, given the lack of reports on this subject. Seventeen stably expressed candidate human miRNAs with suitable signal intensity, $\mathrm{CV}$ across samples and fold-change between groups were shortlisted. Three miRNAs, miR-3937, miR-4257 and miR-1202, were further selected based on the commercial availability of Taqman ${ }^{\circledR}$ real-time PCR assays and the absence of published dysregulation in CRC. While miR-3937 showed a Ct value $>40$, miR-1202 and miR-4257 were detected and confirmed as suitable endogenous controls ( $M$ value $<1.5$ ) by real-time PCR. Fecal miR-1202 and miR-4257 were hence employed as endogenous controls in subsequent real-time PCR analyses.

Validation of real-time PCR method for clinical profiling. Real-time PCR provides a quantitative tool to support clinical profiling of specific miRNA markers identified from the earlier global profiling. Firstly, reaction efficiencies of all amplifications were determined to be between 84.8 and $105.7 \%$, ascertaining minimal presence of PCR inhibitors. $\mathrm{R}^{2}$ coefficients of the calibration curves were $>0.9$. Corresponding NRT controls verified negligible amplification of genomic DNA.

miR-135b, an established fecal marker for CRC $(22,27,28)$, was used as a probe for validating the real-time PCR approach to biomarker confirmation. In accordance with previous reports, fecal miR-135b was significantly upregulated in CRC
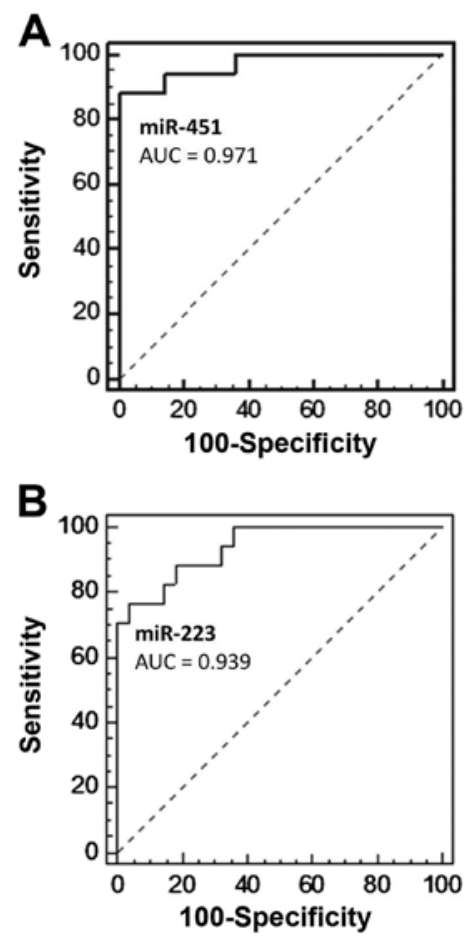

Figure 2. Receiver operator characteristic (ROC) analysis. ROC curves based on logistic regression models (age- and gender-adjusted) using (A) fecal miR-451 and (B) fecal miR-223 for discriminating CRC patients from healthy subjects.

patients compared with healthy subjects. A mean fold-change of $7.25(\mathrm{P}<0.05)$ was observed.

Biomarker confirmation of miR-223 and miR-451 using real-time PCR (phase II). After validating the real-time PCR approach, the CRC-related dysregulation of fecal miR-223 and miR-451 was confirmed in the complete cohort of $17 \mathrm{CRC}$ patients and 28 healthy subjects. From a univariate analysis, the levels of fecal miR-223 and miR-451 were appreciably higher in CRC patients compared to healthy subjects [17.5and 102-fold higher for miR-223 (P<0.001) and miR-451 $(\mathrm{P}<0.001)$, respectively]. Logistic regression analyses with age, gender and fecal miRNAs as independent variables further revealed both fecal miR-223 and miR-451 as potential diagnostic markers $(\mathrm{P}<0.01)$. Receiver operator characteristic (ROC) analyses of the regression models showed areas under the curves (AUCs) of 0.939 (95\% CI, 0.825-0.988) and 0.971 (95\% CI, 0.871-0.998), respectively (Fig. 2). Based on ROC, fecal miR-223 produced a sensitivity of $76.5 \%$ and specificity of $96.4 \%$, while fecal miR-451 yielded a sensitivity of $88.2 \%$ and specificity of $100.0 \%$, in detecting CRC.

Effect of blood in stool on the fecal levels of miRNA. miR-451, miR-223 and miR-135b were present in human blood (Fig. 3), with miR-451 and miR-223 being particularly abundant. Fecal miR-451 level was increased significantly $(\mathrm{P}<0.05)$ by the presence of blood at concentrations as low as $0.1 \mathrm{mg} \mathrm{Hb} / \mathrm{g}$ stool. Fecal miR-223 level was unaffected by occult levels of blood at concentrations up to $1 \mathrm{mg} \mathrm{Hb} / \mathrm{g}$ stool, but was progressively increased $(\mathrm{P}<0.05)$ at $10 \mathrm{mg} \mathrm{Hb} / \mathrm{g}$ stool and beyond. On the contrary, fecal miR-135b remained unchanged at varying levels of blood in stool. 


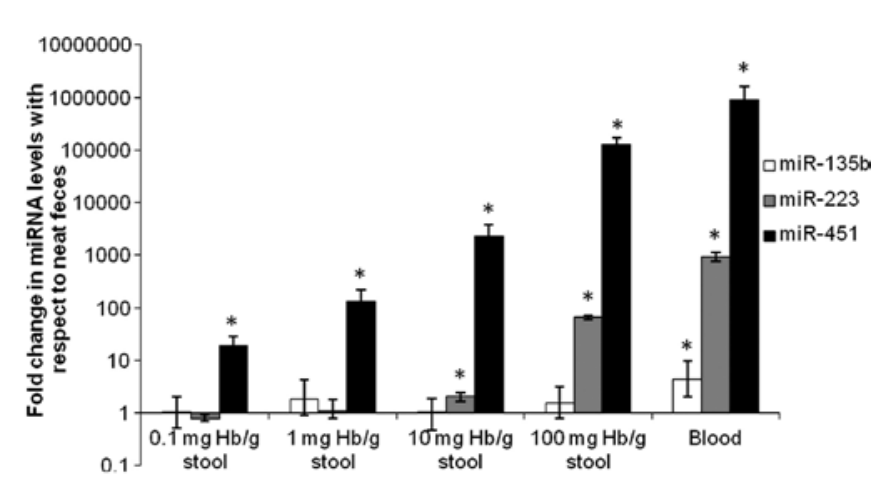

Figure 3. Fold-change (FC) in fecal miRNA levels relative to neat feces at different levels of blood in stool; FC $>1$ indicates higher levels compared to neat feces. Each measurement represents mean fold-change \pm standard error $(\mathrm{n}=3)$. "Statistical significance in FC $(\mathrm{P}<0.05)$.

\section{Discussion}

The search for a reliable, non-invasive fecal-based screening tool has been an ongoing endeavour in the management of CRC. In the present study, we adopted a non-targeted microarray-based approach as a means to uncover novel fecal miRNA markers for detecting $\mathrm{CRC}$ in the Asian population.

Unique fecal and tissue miRNA markers characterizing CRC. The choice of a microarray designed based on the newer Sanger miRBase version 16.0 conferred a broad analytical space for the comprehensive evaluation of fecal and tissue miRNAs. In fecal miRNA profiling, multivariate analysis did not aid in differentiating CRC patients from healthy subjects. The dilution of the disease-related signature was not unexpected considering the plausible inter-individual variations in miRNA expression and the complex nature of the fecal biomatrice. Nevertheless, a univariate analysis unravelled 17 fecal miRNA markers, most of which were previously unreported in the context of CRC. On the other hand, higher expression of fecal miR-223 in CRC patients concurred with Caucasian data $(27,28)$. Taken together, the findings from our global survey of miRNAs confirmed and complemented existing knowledge on fecal miRNA markers of CRC.

Similar to fecal miRNA profiling, both well-characterized and novel markers were elucidated from the global study of miRNAs in paired tumor and normal mucosa. Some differentially expressed miRNAs corresponded to oncomirs well-defined in the pathogenesis of CRC. For example, the miR-17-92 cluster suppresses thrombospondin-1 (Tsp-1) and connective tissue growth factor (CTGF); miR-145 inhibits Myc; and miR-135b targets APC. Tsp-1, CTGF, Myc and APC are regulators along the Wnt signaling pathway known to be altered in CRC (12-20,33). On the other hand, we also uncovered oncomirs not known to correlate with colorectal carcinogenesis, including miR-99a and miR-100 that regulate mTOR in various types of cancer (34-36), as well as newer miRNAs whose functions remain unclear (such as miR-3648).

Notably, unlike a prior Caucasian report (28), these global miRNA aberrations within the tumor were not represented within the fecal miRNA profiles of CRC patients in our study. Osborn and Ahlquist (5) previously defined fecal
Table II. Fold change in tumor tissue $(n=8)$ relative to matched normal mucosae $(n=8)$ from phase I microarray analysis.

\begin{tabular}{lcc}
\hline miRNA & Average fold-change $^{\mathrm{a}}$ & P-value $^{\mathrm{b}}$ \\
\hline miR-451 & 1.26 & 0.708 \\
miR-223 & 1.05 & 0.272 \\
miR-135b & 11.8 & 0.004 \\
\hline
\end{tabular}

${ }^{\text {aF }}$ Fold-change $>1$ indicates higher level in the tumor tissue compared with matched normal mucosae; fold-change $<1$ indicates lower level in the tumor tissue compared with matched normal mucosae. ${ }^{\mathrm{b}} \mathrm{P}$-value from paired t-test.

markers from three sources: exfoliated, secreted and leaked markers. Exfoliated markers are derived from the shedding of colonocytes at the gut luminal surface; secreted markers are emanated from the epithelial cells lining the colonic lumen; and leaked markers are products of disturbed blood or lymph vessels following tumor growth. Our microarray data herein demonstrated that the unique fecal miRNA profile characterizing CRC in Asian patients was possibly associated with processes beyond the direct exfoliation of tumor cells in feces. These complex mechanisms remained unclear, although possible speculations were shedding of normal colonocytes that harbored altered miRNA profiles and the presence of leaked markers from blood in stool of CRC patients. In particular, the former postulation was supported by a recent report that alluded to changes in the miRNA milieu of normal mucosae given its interaction with the adjacent tumor (37). Notably, our observation posed considerable possibilities for future research into the origin of fecal miRNA markers and the multiple features of CRC.

Fecal miR-223 and miR-451 confirmed as clinical markers of $C R C$. Following phase I screening, biomarker confirmation (phase II) was performed on fecal miR-451, a novel and the most differentially expressed fecal marker identified in the microarray analysis. Fecal miR-223 was also included to confirm the observed upregulation that had mirrored Caucasian findings (27,28). From a univariate analysis, fecal miR-223 and miR-451 were noticeably higher in CRC patients compared with healthy subjects, corroborating findings from phase I. Multivariate and ROC analyses further ratified the potential of fecal miR-223 and miR-451 as biomarkers for non-invasive detection of CRC. In future studies, non-CRC subjects with other diseases of the lower gastrointestinal tract, for instance inflammatory bowel disease, could be included to assess the specificity of these fecal markers for CRC.

Recent literature provides plausible targets for these miRNAs but a divided opinion of their influence on oncological phenotypes. For miR-223, it was shown to target tumor suppressor erythrocyte membrane protein band 4.1-like 3 (EPB41L3) in gastric cancer, hence promoting invasion and metastasis (38). Conversely, it suppressed artemin, a tumor promoter, in esophageal carcinoma cells (39) and inhibited cell proliferation through regulation of Forkhead box O-1 (FOXO1) expression in HCT-116 colorectal cancer cell line (40). Hence, 
miR-223 appeared to exert different influence depending on the cancer type. miR-451 regulated LKB1 signaling via direct targeting of calcium-binding protein 39 (CAB39) in glioma cells $(41,42)$. Its overexpression under high glucose conditions permitted unrestrained mTOR activity and thereby promoted cell growth $(41,42)$. However, in an esophageal cancer cell line, high levels of miR-451 downregulated BCL-2, AKT and pAKT and increased apoptosis (43). Similarly, in acute lymphoblastic leukemia, miR-451 suppressed Myc expression (44) while in a DLD-1 colorectal cancer cell line, miR-451 reduced cell proliferation through modulation of macrophage migration inhibitory factor (MIF) expression (45). Therefore, the exact pathological role of miR-451 requires further investigation and contextualization by appropriately considering the influence of their tumor-specific microenvironment. For now, their clinical value mainly rests on their differential expression in patients' fecal material to serve as diagnostics for disease stratification.

Interpretation of fecal miRNA changes considering the influence of blood in stool. As gut bleeding is a clinically prevalent phenomenon of CRC, miRNAs of blood-origin become a pertinent factor in the mechanistic interpretation of fecal miRNA alterations in CRC patients. To the best of our knowledge, this is the first study to investigate the influence of blood in stool on fecal miRNA analysis. Our in vitro data clearly established that blood in the stool affected the levels of three fecal markers, miR-451, miR-223 and miR-135b, to a varying extent. Substantial amounts of miR-451 and miR-223 in the blood were consistent with their reported abundance in erythrocytes and myeloid cells, respectively (30). These notable findings impacted the interpretation of our clinical findings, as discussed below.

Fecal miR-451 level was significantly enriched by the presence of both occult and gross levels of blood. Considering the propensity of gut bleeding in CRC, the data indicated that the observed upregulation of fecal miR-451 in CRC patients may be attributed predominantly to blood in their stool. In other words, it pointed towards the potential role of blood-borne miRNAs as sensitive fecal occult blood markers, or leaked markers, of CRC. On this basis, future studies may be extended to correlating fecal miR-451 with clinical fecal blood levels and comparing its diagnostic sensitivity with existing FOBTs.

Conversely, fecal miR-223 level was unaffected by occult levels of blood up to $1 \mathrm{mg} \mathrm{Hb} / \mathrm{g}$ stool. Based on these data, coupled with the likelihood of gross gut bleeding in some CRC patients, it was postulated that blood in stool accounted partially for the clinical upregulation of fecal miR-223. A separate analysis was conducted on samples documented to contain $<1 \mathrm{mg} \mathrm{Hb} / \mathrm{g}$ stool of blood (8 CRC patients and 10 healthy subjects). This pilot investigation reinforced the pronounced elevation of miR-223 in the feces of CRC patients notwithstanding the absence of blood beyond $1 \mathrm{mg} \mathrm{Hb} / \mathrm{g}$ stool (mean fold-change, 14.6; $\mathrm{P}<0.001$ ). Despite the small cohort size, it underscored the presence of alternative contributors to the upregulation of fecal miR-223 in CRC. Having said that, as we did not observe dysregulation of miR-223 at the tumor level (Table II) in phase I, fecal miR-223 was unlikely a tumorderived exfoliated marker. Collectively, these findings may stimulate future investigations into additional mechanisms for fecal miR-223 perturbation, for instance the shedding of adjacent normal colonocytes and other non-parenchymal cells.
In contrast to fecal miR-451 and miR-223, fecal miR-135b level was impervious to varying amounts of blood, suggesting that blood in stool did not mediate the upregulation of fecal miR-135b in CRC patients; rather, fecal miR-135b was likely a tumor-derived exfoliated marker, as supported by the parallel upregulation at the tumor level (Table II).

In light of the varying degree of influence exerted by the presence of blood in stool on different fecal miRNA markers, we propose that similar experiments be incorporated in the future design of clinical fecal miRNA profiling studies. This approach may also be considered in advancing existing studies where miRNAs highly expressed in blood (including miR-92a in erythrocytes) (30) have been reported as fecal markers of CRC $(22,24,27,28)$. From these efforts, a more comprehensive insight into the alterations of potential fecal miRNA markers could be gleaned.

In conclusion, the present study highlighted the utility of a holistic miRNA screening approach in elucidating potential diagnostic markers of CRC in the Asian population. Fecal miR-223 and miR-451 were further confirmed as biomarkers that may facilitate the non-invasive screening of CRC. It also illustrated the importance of delineating the influence of blood in stool and integrating these findings during the interpretation of clinical fecal miRNA data.

\section{Acknowledgements}

The authors are grateful to Ms. Jiamin Koh, Dr Ming Hian Kam, Ms. Pauline Ngiik Hung Wong and the Department of Colorectal Surgery at Singapore General Hospital for their support in subject recruitment, as well as Ms. Elya, Ms. Michelle Shu Mei Lo, Ms. Wei Lin Goh and Ms. Grace Yu Hui Wong for their technical assistance and retrieval of the clinicopathological data. This project was funded by the Singapore Ministry of Education's (MOE) Academic Research Grants R -148-000-133-112 (HKH) and R-148-000-135-112 (ECYC). LCP was supported by the NUS President Graduate Fellowship.

\section{References}

1. Ferlay J, Shin HR, Bray F, Forman D, Mathers C and Parkin DM: GLOBOCAN 2008 v2.0, Cancer Incidence and Mortality Worldwide: IARC CancerBase No. 10 (Internet). International Agency for Research on Cancer, Lyon, 2010. http://globocan.iarc. fr (access date: 11/06/2013).

2. Moghimi-Dehkordi B and Safaee A: An overview of colorectal cancer survival rates and prognosis in Asia. World J Gastrointest Oncol 4: 71-75, 2012.

3. Sung JJ, Lau JY, Goh KL and Leung WK: Increasing incidence of colorectal cancer in Asia: implications for screening. Lancet Oncol 6: 871-876, 2005.

4. Ransohoff DF and Sandler RS: Clinical practice. Screening for colorectal cancer. N Engl J Med 346: 40-44, 2002.

5. Osborn NK and Ahlquist DA: Stool screening for colorectal cancer: molecular approaches. Gastroenterology 128: 192-206, 2005.

6. Takai T, Kanaoka S, Yoshida K, et al: Fecal cyclooxygenase 2 plus matrix metalloproteinase $7 \mathrm{mRNA}$ assays as a marker for colorectal cancer screening. Cancer Epidemiol Biomarkers Prev 18: 1888-1893, 2009.

7. Bartel DP: MicroRNAs: genomics, biogenesis, mechanism, and function. Cell 116: 281-297, 2004.

8. Hwang HW and Mendell JT: MicroRNAs in cell proliferation, cell death, and tumorigenesis. Br J Cancer 94: 776-780, 2006.

9. Calin GA and Croce CM: MicroRNA signatures in human cancers. Nat Rev Cancer 6: 857-866, 2006. 
10. Kong YW, Ferland-McCollough D, Jackson TJ and Bushell M: microRNAs in cancer management. Lancet Oncol 13: e249-e258, 2012.

11. Esquela-Kerscher A and Slack FJ: Oncomirs - microRNAs with a role in cancer. Nat Rev Cancer 6: 259-269, 2006

12. Motoyama K, Inoue H, Takatsuno Y, et al: Over- and underexpressed microRNAs in human colorectal cancer. Int $\mathrm{J}$ Oncol 34: 1069-1075, 2009.

13. Bandres E, Cubedo E, Agirre X, et al: Identification by Real-time PCR of 13 mature microRNAs differentially expressed in colorectal cancer and non-tumoral tissues. Mol Cancer 5: 29, 2006.

14. Wang CJ, Zhou ZG, Wang L, et al: Clinicopathological significance of microRNA-31, -143 and -145 expression in colorectal cancer. Dis Markers 26: 27-34, 2009.

15. Chen Y, Song Y, Wang Z, et al: Altered expression of miR-148a and miR-152 in gastrointestinal cancers and its clinical significance. J Gastrointest Surg 14: 1170-1179, 2010.

16. Slaby O, Svoboda M, Fabian P, et al: Altered expression of miR-21, miR-31, miR-143 and miR-145 is related to clinicopathologic features of colorectal cancer. Oncology 72: 397-402, 2007.

17. Hamfjord J, Stangeland AM, Hughes T, et al: Differential expression of miRNAs in colorectal cancer: comparison of paired tumor tissue and adjacent normal mucosa using high-throughput sequencing. PLoS One 7: e34150, 2012.

18. Faltejskova P, Svoboda M, Srutova K, et al: Identification and functional screening of microRNAs highly deregulated in colorectal cancer. J Cell Mol Med 16: 2655-2666, 2012.

19. Xu XM, Qian JC, Deng ZL, et al: Expression of miR-21, miR-31, miR-96 and miR-135b is correlated with the clinical parameters of colorectal cancer. Oncol Lett 4: 339-345, 2012.

20. Mazeh H, Mizrahi I, Ilyayev N, et al: The diagnostic and prognostic role of microRNA in colorectal cancer - a comprehensive review. J Cancer 4: 281-295, 2013.

21. Ahmed FE, Jeffries CD, Vos PW, et al: Diagnostic microRNA markers for screening sporadic human colon cancer and active ulcerative colitis in stool and tissue. Cancer Genomics Proteomics 6: 281-295, 2009.

22. Koga Y, Yasunaga M, Takahashi A, et al: MicroRNA expression profiling of exfoliated colonocytes isolated from feces for colorectal cancer screening. Cancer Prev Res (Phila) 3: 1435-1442, 2010.

23. Link A, Balaguer F, Shen Y, et al: Fecal microRNAs as novel biomarkers for colon cancer screening. Cancer Epidemiol Biomarkers Prev 19: 1766-1774, 2010.

24. Wu CW, Ng SS, Dong YJ, et al: Detection of miR-92a and miR-21 in stool samples as potential screening biomarkers for colorectal cancer and polyps. Gut 61: 739-745, 2011.

25. Yamazaki N, Koga Y, Yamamoto S, et al: Application of the fecal microRNA test to the residuum from the fecal occult blood test Jpn J Clin Oncol 43: 726-733, 2013.

26. Li JM, Zhao RH, Li ST, et al: Down-regulation of fecal miR-143 and miR-145 as potential markers for colorectal cancer. Saudi Med J 33: 24-29, 2012.

27. Kalimutho M,Del Vecchio Blanco G,DiCecilia S, et al: Differential expression of miR-144* as a novel fecal-based diagnostic marker for colorectal cancer. J Gastroenterol 46: 1391-1402, 2011.

28. Ahmed FE, Ahmed NC, Vos PW, et al: Diagnostic microRNA markers to screen for sporadic human colon cancer in stool: I. Proof of principle. Cancer Genomics Proteomics 10: 93-113, 2013.
29. Mao X, Yu Y, Boyd LK, et al: Distinct genomic alterations in prostate cancers in Chinese and Western populations suggest alternative pathways of prostate carcinogenesis. Cancer Res 70: 5207-5212, 2010

30. Pritchard CC, Kroh E, Wood B, et al: Blood cell origin of circulating microRNAs: a cautionary note for cancer biomarker studies. Cancer Prev Res (Phila) 5: 492-497, 2012.

31. Kirschner MB, Kao SC, Edelman JJ, et al: Haemolysis during sample preparation alters microRNA content of plasma. PLoS One 6: e24145, 2011

32. Vandesompele J, De Preter K, Pattyn F, et al: Accurate normalization of real-time quantitative RT-PCR data by geometric averaging of multiple internal control genes. Genome Biol 3: RESEARCH0034, 2002.

33. Slaby O, Svoboda M, Michalek J and Vyzula R: MicroRNAs in colorectal cancer: translation of molecular biology into clinical application. Mol Cancer 8: 102, 2009.

34. Sun J, Chen Z, Tan X, et al: MicroRNA-99a/100 promotes apoptosis by targeting mTOR in human esophageal squamous cell carcinoma. Med Oncol 30: 411, 2013.

35. Torres A, Torres K, Pesci A, et al: Deregulation of miR-100, miR-99a and miR-199b in tissues and plasma coexists with increased expression of mTOR kinase in endometrioid endometrial carcinoma. BMC Cancer 12: 369, 2012.

36. Chen Z, Jin Y, Yu D, et al: Down-regulation of the microRNA-99 family members in head and neck squamous cell carcinoma. Oral Oncol 48: 686-691, 2012.

37. Huang ZM, Yang J, Shen XY, et al: MicroRNA expression profile in non-cancerous colonic tissue associated with lymph node metastasis of colon cancer. J Dig Dis 10: 188-194, 2009.

38. Li X, Zhang Y, Zhang H, et al: miRNA-223 promotes gastric cancer invasion and metastasis by targeting tumor suppressor EPB41L3. Mol Cancer Res: 824-833, 2011.

39. Li S, Li Z, Guo F, et al: miR-223 regulates migration and invasion by targeting Artemin in human esophageal carcinoma. J Biomed Sci 18: 24, 2011.

40. Wu L, Li H, Jia CY, et al: MicroRNA-223 regulates FOXO1 expression and cell proliferation. FEBS Lett 586: 1038-1043, 2012

41. Godlewski J, Bronisz A, Nowicki MO, Chiocca EA and Lawler S microRNA-451: a conditional switch controlling glioma cell proliferation and migration. Cell Cycle 9: 2742-2748, 2010.

42. Godlewski J, Nowicki MO, Bronisz A, et al: MicroRNA-451 regulates LKB1/AMPK signaling and allows adaptation to metabolic stress in glioma cells. Mol Cell 37: 620-632, 2010.

43. Wang T, Zang WQ, Li M, Wang N, Zheng YL and Zhao GQ: Effect of miR-451 on the biological behavior of the esophageal carcinoma cell line EC9706. Digest Dis Sci 58: 706-714, 2013.

44. Molinari F, Felicioni L, Buscarino M, et al: Increased detection sensitivity for KRAS mutations enhances the prediction of anti-EGFR monoclonal antibody resistance in metastatic colorectal cancer. Clin Cancer Res 17: 4901-4914, 2011.

45. Bandres E, Bitarte N, Arias F, et al: microRNA-451 regulates macrophage migration inhibitory factor production and proliferation of gastrointestinal cancer cells. Clin Cancer Res 15: 2281-2290, 2009. 